\title{
Quantitative analysis of 2-hydroxyglutarate as a controversial oncometabolite in malignant gliomas
}

\author{
P. BYSTRICKY ${ }^{1, *}$, I. KASUBOVA ${ }^{1}$, R. RICHTEROVA ${ }^{2}$, Z. LASABOVA ${ }^{3}$, B. KOLAROVSZKI ${ }^{2}$ \\ ${ }^{1}$ Biomedical Center Martin, Jessenius Faculty of Medicine in Martin, Comenius University in Bratislava, Martin, Slovakia; ${ }^{2}$ Department of \\ Neurosurgery, University Hospital Martin and Jessenius Faculty of Medicine in Martin, Comenius University in Bratislava, Martin, Slovakia; \\ ${ }^{3}$ Department of Molecular Biology and Genomics, Jessenius Faculty of Medicine in Martin, Comenius University in Bratislava, Martin, Slovakia.
}

${ }^{*}$ Correspondence: peter.bystricky@uniba.sk

Received March 2, 2020 / Accepted July 9, 2020

\begin{abstract}
There is a great effort to connect the accumulation of 2-hydroxyglutarate (2-HG) oncometabolite with cellular oncoepigenetic status and subsequently predict the prognoses of glioma patients. In this observational study, the concentrations of D- and L- 2-HG were determined in 57 tumor tissue samples of glioma patients $(\mathrm{n}=57)$ WHO grade I through IV (astrocytoma, oligodendroglioma, secondary glioblastoma, and glioblastoma multiforme) in vitro. Also, genetic mutation status on isocitrate dehydrogenase 1 and 2 (IDH 1/2) was determined from these samples. The objective of this study was to confirm or to reject the hypothesis of the direct correlation of 2-HG concentration in tumor tissue and the results from IDH 1/IDH 2 point mutation analyses. The concentrations of 2-HG were quantified using high sensitive HPLC and Q-TOF HRMS spectrometer setup. Concurrently, the genetic mutation analyses of both IDH 1 (cytosolic) and IDH 2 (mitochondrial) were performed by the isolation of tumor tissue DNA, PCR amplification, and subsequent Sanger forward sequencing. Our results indicate that there is no definite correlation between the two as we identified cases of glioma tumors with significantly increased concentration of one or both L- and D- 2-HG but no IDH 1/2 mutations (44\% 2-HG positive cases).
\end{abstract}

Key words: 2-hydroxyglutarate, Krebs cycle, oncometabolite, malignant gliomas, WHO grade, astrocytoma, oligodendroglioma, glioblastoma multiforme, IDH mutations

Recently, single point mutations in cytosolic isocitrate dehydrogenase 1 and mitochondrial isocitrate dehydrogenase 2 (IDH 1/2) have been discovered [1]. These mutations have been found mostly in lower WHO grades gliomas (LGG, WHO grades I through III) and to a lesser extent in high-grade glioma (WHO grade IV, e.g. glioblastoma multiforme) [2]. These mutations can be heterozygous or very rarely homozygous [3]. The reported incidence of IDH $1 / 2$ mutations in low-grade gliomas is about $70 \%$, in glioblastoma multiforme about $7-10 \%$ [4]. These mutations are present also in acute myeloid leukemia [5], chondrosarcoma [6], cholangiocarcinoma [7], and T-cell lymphoma [8]. Subsequent identification of 2-hydroxyglutarate (2-HG), an oncometabolite, as a suggested direct consequence of these mutations has provided new insights into the onset of this disease [9]. 2-HG has a chiral center, so it has two isomers - (R) and (S) also called D- and L- isomers. In acidic conditions, it forms an internal lactone (5-oxotetrahydrofuran2 -carboxylic acid) [10]. The IDH 1/2 mutations occur in both enzymes in a single amino acid that is localized in the active site. Structural studies show that when arginine 132 is mutated to histidine in IDH 1 , amino acids at the active site are shifted and produce 2-HG instead of 2-oxoglutarate. It is the same case for arginine 172 to histidine in IDH 2 [11]. It is believed that this results in the loss of the ability of these enzymes to catalyze the conversion of isocitrate to 2-oxoglutarate (2-OG). Moreover, these cancer-associated IDH 1/2 mutations have been shown to catalyze the enzyme's ability to reduce 2 -oxoglutarate to $\mathrm{D}$-2-hydroxyglutarate (D-2-HG) in the nicotinamide adenine dinucleotide phosphate $\left(\mathrm{NADP}^{+}\right)$ dependent manner as a part of a cycle of the tricarboxylic acids (Krebs cycle) [12]. Accumulation of high concentrations of $2-\mathrm{HG}$ in cells with IDH $1 / 2$ mutation has given rise to the development of $2-\mathrm{HG}$ as a clinical oncomarker. Extensive molecular studies of lower-grade gliomas in adults (astrocytoma, oligodendroglioma, and secondary glioblastoma) defined the IDH mutation also as very important in the determination of the patient's prognosis. Some authors believe that the mutated IDH 1/2 form 2-OG just like wild type enzymes but they also convert 2-OG to 2-HG [12]. 
Human malignant gliomas that contain IDH $1 / 2$ mutations should have a dramatically elevated concentration of $2-\mathrm{HG}$ in vivo which contributes to the malignant nature of the tumor. The controversy of $2-\mathrm{HG}$ is that in the case of glioblastoma when IDH $1 / 2$ mutation is detected it lowers the WHO grade and changes the diagnosis to secondary glioblastoma and the prognosis of the patient is prolonged [13]. This is the main point of the controversy.

The ${ }^{13} \mathrm{C}$ nuclear magnetic resonance (NMR) spectroscopy was suggested as a method for patient tumor extracts analyses [4]. However, only helium cooled NMR microprobes provide sufficient sensitivity to detect the natural abundance ${ }^{13} \mathrm{C} 2-\mathrm{HG}$ from tumor samples [14]. In addition, it is well known that 2-HG can be detected by high performance liquid chromatography - mass spectrometry (HPLCMS) in vitro and by localized MRS in vivo [15]. HPLC chromatography is used for the separation of molecular species and MS is used as an extremely sensitive and highly selective detector. This highly sensitive HPLC-MS quantification of 2-HG has not yet been used in clinical practice. One reason for this is the fact that expensive and technologically demanding instruments are required. In the case of an in vitro assay, a high-performance liquid chromatograph coupled to a mass spectrometer enabling the acquisition of MS-MS $\left(\mathrm{MS}^{2}\right)$ at least at low resolution is required. Such in vitro quantification can be considered a gold standard. There are less reliable immunohistological staining for IDH 1 mutations only and D-2-HG colorimetric assay, which is also insensitive and unreliable. The objective of this study was to confirm or reject the hypothesis of the direct correlation of $2-\mathrm{HG}$ concentration in tumor tissue and the results from IDH 1 / IDH 2 genetic point mutation analyses. The initial selection of patients was done based on the histology findings. In addition, these results from the histology and immunohistochemistry for IDH 1 mutations were correlated to genetic mutational analyses (and of course in vitro 2-HG concentrations). For this study, a high-resolution mass spectrometer (HRMS) capable of $\mathrm{MS}^{2}$ acquisition was used for 2-HG detection and quantification.

This sensitive and reliable method has the potential to be helpful in diagnoses and prognoses of the glioma patients. Also, the relationship between $2-\mathrm{HG}$ concentration and IDH $1 / 2$ mutations could contribute to the understanding of tumor multiple metabolic pathways.

\section{Patients and methods}

Sample selection. 57 tumor biopsy tissue samples from Slovak glioma patients were available and selected for the study. The patients were mostly from northern and central Slovakia. The accepted diagnoses were glioma WHO grades I through IV e.g. WHO grade I: pilocytic astrocytoma, subependymal giant cell astrocytoma; WHO grade II: low-grade (fibrillary) astrocytoma, pleomorphic xanthoastrocytoma, oligodendroglioma, mixed oligoastrocytoma; WHO grade
III: anaplastic astrocytoma; WHO grade IV: secondary glioblastoma multiforme (GBM) and primary glioblastoma multiforme. The patients had the aforementioned diagnoses confirmed from the histological slides by the presence of distinctive histological features. The histological slides were made from tissue post-surgical removal of part of their tumor.

Ethics approval and consent to participate. The involvement of the patients in this study was approved by the Ethics Committee of the Jessenius Faculty of Medicine in Martin (protocol no. EK 144/2018). All patients signed written informed consent prior to the collection of biopsy tissue samples used in this study.

2-HG extraction from tumor tissue. The 2-HG extraction procedure was adopted from Struys et al. [16] with minor modifications. We used water ice for cooling the samples and we homogenized them in the extraction mixture on TissueLyser LT (QIAGEN) with small stainless-steel ball (QIAGEN) in the tube. After homogenization, both extracts were combined in a $2 \mathrm{ml}$ glass screw cap tube and they were open dried in a flow box.

DATAN derivatization. In order to separate 2-HG on the $\mathrm{C} 18$ column, it needs to be retained on the column to an adequate degree. Therefore, the 2-HG (a small molecule) needs to be enlarged [16]. In addition, this derivatization should provide separation of L- and D- isomers [17]. This is accomplished by derivatization with di-O-acetyl-L-tartaric anhydride (DATAN) [16]. It also prevents the formation of internal lactone mentioned above. DATAN derivatization was performed according to procedures in Collins et al. [18] but the reaction time was extended to $2-3 \mathrm{~h}$. In order to remove residual acetic acid completely, $0.5 \mathrm{ml}$ of LC-MS grade water was added and the samples were lyophilized.

Analysis of 2-HG DATAN derivatives, HPLC conditions, MS setup. Tissue extracts were dissolved in $20 \mu \mathrm{ml}$ of $2 \mathrm{mM}$ ammonium formate, $\mathrm{pH} 3.60$ along with both L- and D-2-HG standard sets. We used Phenomenex Kinetex ${ }^{\oplus}$ Polar C18 3.0 mm ID × 150 mm L, $2.6 \mu$ m particle size HPLC column, and following eluents: A) $2 \mathrm{mM}$ ammonium format, $\mathrm{pH} 3.60, \mathrm{~B}$ ) acetonitrile (ACN). The gradient program was as follows: $0-0.5 \mathrm{~min} 100 \% \mathrm{~A}, 0.5-8$ min linear gradient to $3.5 \% \mathrm{ACN}, 8-16$ min linear gradient to $50 \% \mathrm{ACN}, 3 \mathrm{~min} 100 \% \mathrm{ACN}$ column wash, flow rate $0.30 \mathrm{ml} / \mathrm{min}$ (typical backpressure $270 \mathrm{bar}$ at these conditions). The standards and samples were injected into HPLC in a so-called bracketing fashion (2 calibration set injections: before and after tissue extract samples). Detection was done on Bruker Q-TOF Impact II HRMS spectrometer (Karlsruhe, Germany) in negative MRM mode. MRM reactions were set up according to $363.05->147.03 \mathrm{~m} / \mathrm{z}$ and $366.06->150.05 \mathrm{~m} / \mathrm{z}$ (triple deuterated IS). Acquired data were analyzed by Bruker Quant Analysis software. From MS data L-and D-2-HG peaks in EIC chromatograms set for $363.05 \pm 0.01 \mathrm{~m} / \mathrm{z}$ were integrated and plotted separately for the L- and D- isomers (EIC chromatograms were set for $366.06 \pm 0.01 \mathrm{~m} / \mathrm{z}$ for IS) as calibration curves 
in the software. Also, from MS/MS data L- and D- 2-HG peaks in EIC chromatograms set for $147.03 \pm 0.01 \mathrm{~m} / \mathrm{z}$ (EIC chromatograms were set for $150.05 \pm 0.01 \mathrm{~m} / \mathrm{z}$ for IS).

Genetic mutation analyses. Genetic mutation analyses were performed on isolated DNA from tumor tissues. About $20 \mathrm{mg}$ of tissue was used for DNA isolation. DNA was isolated from tumor tissue using QIAamp Fast DNA Tissue Kit (QIAGEN, GmbH, Hilden, Germany) according to the manufacturer's protocol. DNA concentration was measured on a Qubit ${ }^{\oplus}$ 2.0 Fluorimeter (Invitrogen; Thermo Fisher Scientific, Inc., Waltham, MA, USA) and diluted (if needed) to working solutions at a concentration of 100 $\mathrm{ng} / \mu \mathrm{l}$ and stored at $20^{\circ} \mathrm{C}$. The presence of variants in the exon 4 of the IDH 1 gene and in the exon 4 of the IDH 2 gene was monitored by Sanger sequencing using an $\mathrm{ABI}$ 3500 (Applied Biosystems; Thermo Fisher Scientific, Inc.). We used a standard polymerase chain reaction (PCR) to amplify all exon 4 for both genes. PCR was performed using a GeneAmp PCR System 9700 instrument (Applied Biosystems; Thermo Fisher Scientific, Inc.) and amplified with specific primers. The primer sequences were: IDH 1 Forward 5`-GCCAGTGCTAAAACTTGGCAG-3`; IDH 1 Reverse 5 '-TCAATTTCATACCTTGCTTAATGGG-3 '; IDH 2 Forward 5'-GCAGACTCCAGAGCCCACAC-3`; IDH 2 Reverse 5 '-TGCCATCTTTTGGGGTGAAG-3 '.

PCR conditions were set to a total volume of $20 \mu$ l. The primer annealing temperature for both PCR amplifications was optimized to $60^{\circ} \mathrm{C}$. The success of PCR amplifications was verified by agarose electrophoresis in a $1.75 \%$ agarose gel. The first purification of PCR products was performed using a NucleoSpin Gel and PCR clean up kit (Machery-Nagel $\mathrm{GmbH}$, Düren, Germany) according to the manufacturer's protocol. By this purification, we degraded single-stranded primer residues and hydrolyzed dNTPs residues. Subsequently, we prepared a sequencing reaction using BigDye Terminator v1.1 Cycle Sequencing kit (Thermo Fisher Scientific, Inc.). The reaction was run in a total volume of $10 \mu \mathrm{l}$. The non-annealing (hybridization) temperatures were consistent with the annealing temperature of the primers during the PCR reaction. SigmaSpin Post Reaction Clean Up (Sigma Aldrich; Merck KGaA, Darmstadt, Germany) was used for the purification after the sequencing reaction according to the information provided by the manufacturer. We used the Sanger sequencing method for sample sequencing. Plates with samples were prepared for analysis. After PCR clean up, $3 \mu \mathrm{l}$ of the purified product was added to $12 \mu \mathrm{Hi} \mathrm{Di}^{\text {Ts }}$ formamide (Applied Biosystems; Thermo Fisher Scientific, Inc.). Capillary electrophoresis was performed in a POP-7 polymer (Applied Biosystems; Thermo Fisher Scientific, Inc.) with a $50 \mathrm{~cm}$ capillary on an ABI 3500 genetic analyzer (Applied Biosystems, Thermo Fisher Scientific, Inc). Sequencing data obtained were evaluated and compared with the reference sequence of the analyzed regions using Chromas Pro Ver. 3.1 software (Technelysium Pty Ltd., South Brisbane, QLD, Australia). The gene sequences obtained from the ENSEMBL database (https://www.ensembl.org/index.html) served as reference sequences.

\section{Results}

We utilized and developed the method of quantitative analysis of 2-HG by the technique of high-performance liquid chromatography with high-resolution mass spectrometry. We managed to separate L- and D-isomers in our instrumental setup. It was found that the separation was very sensitive to the $\mathrm{pH}$ of the buffer (3.60) and the $\mathrm{pH}$ needed to be checked regularly or freshly prepared. The results are summarized in Table 1. An example of extracted ion chromatogram from patient with highest L- and D-2-HG concentrations (43-year-old male astrocytoma patient with heterozygous IDH 1 mutation) is in Figure 1A. A representative MS spectrum from chromatogram at the retention time of $10.70 \mathrm{~min}$ is shown in Figure 1B.

As Table 1 shows, out of the 6 astrocytoma patients, only 3 were positive for 2 -HG (only $50 \%$ ), out of the 3 oligodendroglioma patients, 2 were positive for 2 -HG (70\%), and 1 secondary glioblastoma was not positive for 2-HG.

Moreover, out of the 44 primary glioblastomas, only 3 were positive for $2-\mathrm{HG}(7 \%)$. It is however clear that we have statistical sets for astrocytomas, oligodendrogliomas, and secondary glioblastomas that are still too small. Genetic analyses for IDH $1 / 2$ mutations revealed that all IDH 1 detected mutations were of R132H type (CGT -> CAT in the sequence) and all of the heterozygous type. On the other hand, a single detected IDH 2 mutation was of rare $\mathrm{R} 172 \mathrm{~K}$ mutation (AGG -> ACG). Examples of heterozygous mutations are in Figure $1 \mathrm{C}$ and $1 \mathrm{D}$ for IDH 1 and IDH 2 mutation, respectively. Both, genetic mutation and HPLC-HRMS analyses revealed that only $36 \%$ of grade II and III gliomas (astrocytoma, oligodendroglioma, secondary glioblastoma) were 2-HG positive (either D-2-HG or L- and D-2-HG positive). All available immunohistochemistry results for IDH 1 mutations correlated very well with the results from HPLC-HRMS analyses. Notably, a 43-yearold male had astrocytoma diagnosed based on histology slide. This diagnosis was followed by surgical resection of the tumor. Furthermore, immunohistochemistry revealed IDH 1 mutation and genetic mutation analysis revealed heterozygous IDH $1 \mathrm{R} 132 \mathrm{H}$ mutation. Moreover, HPLCHRMS analysis for 2-HG revealed very high concentrations of both L- and D-2-HG (which are both products of mutated IDH 1). This was a textbook example of an astrocytoma case with IDH 1 mutation where all data correlate with each other.

\section{Discussion}

The patients were diagnosed by radiologists, surgeons, and histologists, however they all gave only basic diagnosis (astrocytoma, oligodendroglioma, secondary glioblastoma, and glioblastoma multiforme). Only in one case, they reported a 
Table 1. Glioma patients including their diagnosis, IDH 1/2 mutation analysis, and concentration of 2-HG in the tissue.

\begin{tabular}{|c|c|c|c|c|c|c|}
\hline Sex & Age & $\begin{array}{l}\text { Diagnosis from histology, immu- } \\
\text { nohistochemistry analysis result }\end{array}$ & $\begin{array}{l}\text { IDH } 1 / 2 \text { mutation } \\
\text { analysis }\end{array}$ & $\begin{array}{c}\text { Concentration of } \\
\text { D-2-HG [pmol/mg } \\
\text { tissue] }\end{array}$ & $\begin{array}{c}\text { Concentration of } \\
\text { L-2-HG [pmol/mg } \\
\text { tissue] }\end{array}$ & $\begin{array}{l}\text { Total 2-HG Concen- } \\
\text { tration (D + L 2-HG) } \\
\text { [pmol/mg tissue] }\end{array}$ \\
\hline male & 43 & astrocytoma, IDH 1 mut. positive & $\begin{array}{l}\text { IDH } 1(\mathrm{R} 132 \mathrm{H}) \\
\text { heterozygous }\end{array}$ & 6730 & 629 & 7359 \\
\hline male & 49 & astrocytoma & $\begin{array}{l}\text { IDH } 1(\mathrm{R} 132 \mathrm{H}) \\
\text { heterozygous }\end{array}$ & 566 & 6 & 572 \\
\hline male & 40 & astrocytoma & $\begin{array}{l}\text { IDH } 1(\mathrm{R} 132 \mathrm{H}) \\
\text { heterozygous }\end{array}$ & 168 & 0 & 168 \\
\hline female & 63 & glioblastoma multiforme & negative & 156 & 141 & 297 \\
\hline male & 48 & oligodendroglioma & $\begin{array}{l}\text { IDH } 2 \text { (R172K) } \\
\text { heterozygous }\end{array}$ & 136 & 0 & 136 \\
\hline male & 64 & glioblastoma multiforme & negative & 2 & 64 & 66 \\
\hline female & 59 & oligodendroglioma & $\begin{array}{l}\text { IDH } 1(\mathrm{R} 132 \mathrm{H}) \\
\text { heterozygous }\end{array}$ & 40 & 0 & 40 \\
\hline female & 69 & glioblastoma multiforme & negative & 10 & 32 & 42 \\
\hline male & 51 & $\begin{array}{l}\text { metastases from melanoma, mar- } \\
\text { ginally IDH } 1 \text { mut. positive }\end{array}$ & negative & 14 & 0 & 14 \\
\hline 17 females & $40-79$ & glioblastoma multiforme & & & & \\
\hline 25 males & $52-72$ & glioblastoma multiforme & & & & \\
\hline female & 62 & secondary glioblastoma & & & & \\
\hline male & 42 & astrocytoma & negative & $0-3$ & $0-3$ & $0-6$ \\
\hline male & 68 & oligodendroglioma & negative & (negative) & (negative) & (negative) \\
\hline male & 62 & anaplastic astrocytoma & & & & \\
\hline male & 46 & secondary glioblastoma & & & & \\
\hline male & 65 & astrocytoma & & & & \\
\hline
\end{tabular}

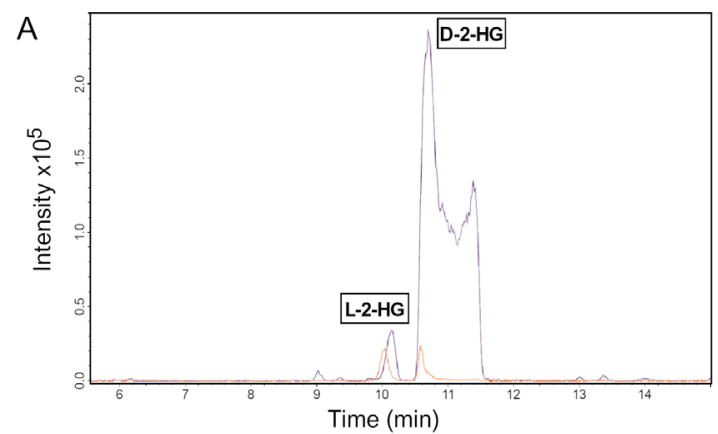

B

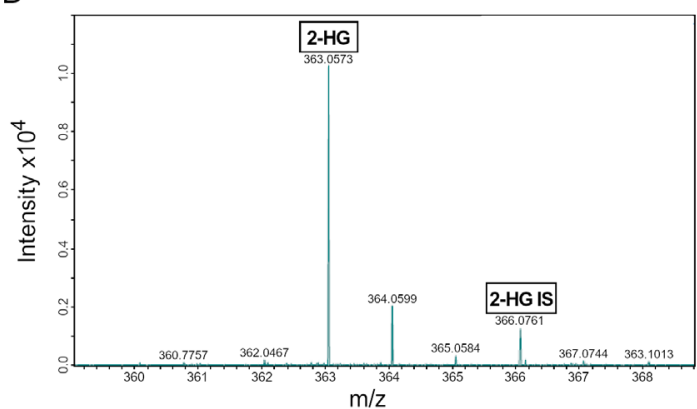

C

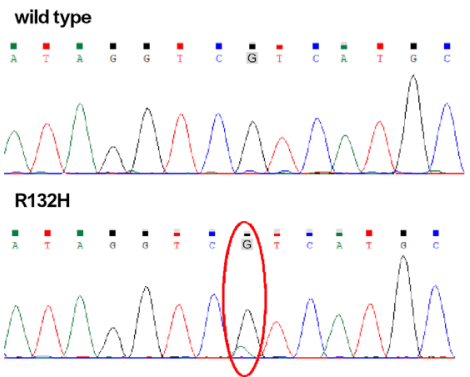

D

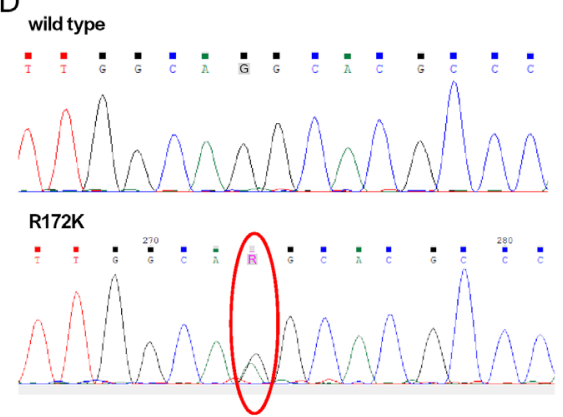

Figure 1. A) Typical extracted ion chromatogram at $363.06 \pm 0.01 \mathrm{~m} / \mathrm{z}(2-\mathrm{HG}$ DATAN derivative in negative mode). Data are from 43 -year-old male astrocytoma patient with heterozygous IDH 1 mutation and the highest levels of both L- and D-2-HG concentrations. Orange trace is 2-HG triple deuterated internal standard and violet trace is natural abundance 2-HG from the sample. Retention times are $10.15 \mathrm{~min}$ for L-2-HG and $10.70 \mathrm{~min}$ for D-2-HG. B) MS spectrum from chromatogram in 1A at 10.70 min includes natural abundance 2-HG sample peaks $(363.0573,364.0599$, and 365.0584 $\mathrm{m} / \mathrm{z}$ ) and 2-HG triple deuterated internal standard (IS) peaks (366.0761,367.0744, and 368.1013). C and D) Results of genetic mutation analysis of IDH $1(\mathrm{R} 132 \mathrm{H})$ and IDH $2(\mathrm{R} 172 \mathrm{~K})$ as well as wild unmutated wild types. In panel C) there are data from a 42-year-old male with astrocytoma and no IDH mutation and a 40-year-old male with IDH 1 heterozygous mutation. Data from a 68-year-old male with oligodendroglioma and no IDH mutation and one and only 48-year-old male with IDH 2 heterozygous mutation are shown in panel D. 
more specific diagnosis (anaplastic astrocytoma). Nevertheless, the results revealed one important fact: in four cases we detected elevated concentration of $2-\mathrm{HG}$ but no mutation in either IDH 1 or IDH 2. One of these cases was metastases to the brain from melanoma and although the concentration of D-2-HG was marginally elevated, it was by very little. The other three cases were more significant. The elevated concentration of 2-HG in these cases could be explained by the fact that healthy individuals (without any glioma) also have a small amount of 2-HG in the brain tissue. The search of the literature yields an explanation: the cause is that L-2-HG is also synthesized by wild type malate dehydrogenase and D-2-HG is synthesized by wild type hydroxyacid or oxoacid transhydrogenases in healthy individuals [18]. Others explain this state as a consequence of elevated mitochondrial glutaminase activation. This increases the production (flux) of glutamate and in turn ketoglutarate and then to 2-HG [19]. Nevertheless, in healthy individuals L- and D- 2-HG are consumed by L- and D-2-hydroxyglutarate dehydrogenases [18]. Therefore in health, there is a balance between this catabolism and anabolism. The hypothesis here is that this balance then must be disturbed as a part of the tumor metabolism in these three patients with no IDH 1/2 mutations. Increased levels of Land/or D-2-HG have an effect on DNA and cause DNA and histone hypermethylation, HIF stabilization with pseudohypoxia response, and epithelial mesenchymal transition [16]. The future proceedings with this research are to quantify 2-HG in samples of genetically confirmed mutation on IDH $1 / 2$ in the tumor tissue sample, plasma, and urine by HPLCHRMS. From these data, we want to map the distribution of this oncometabolite in the body and body fluids. In addition, we want to determine the concentration of 2-HG directly in the tumor in vivo by MRS in the same patients to confirm the correlation between HPLC-HRMS in vitro with in vivo MRS 2-HG concentration. Therefore, only a complex picture of $2-\mathrm{HG}$ distribution in the body could revealed the consequences of altered metabolic pathways at the onset of these neuro-oncological diseases.

Acknowledgments: The authors would like to thank prof. Peter Račay for the initial idea of the study, Dr. Mária Škereňová for helpful discussions and Mgr. Simona Holubčíková for technical assistance. This work was supported by the projects "Biomedical Center Martin”, ITMS code: 6220220187 co-financed from EU sources, "Center of Excellence for Research in Personalized Therapy (CEVYPET)", ITMS code: 26220120053 supported by the Operational Programme Research and Innovation funded by the ERDF and project no. 2018/13-UKMT-9 from Ministry of Health of the Slovak Republic.

\section{References}

[1] PARSONS DW, JONES S, ZHANG X, LIN JC-H, LEARY RJ et al. An Integrated Genomic Analysis of Human Glioblastoma Multiforme. Science 2008; 321: 1807-1812. https://doi. org/10.1126/science.1164382
[2] DANG L, WHITE DW, GROSS S, BENNETT BD, BITTINGER MA et al. Cancer-associated IDH1 mutations produce 2-hydroxyglutarate. Nature 2009; 462: 739-744. https:// doi.org/10.1038/nature08617

[3] SINGH A, GURAV M, DHANAVADE S, SHETTY O, EPARI S. Diffuse glioma - Rare homozygous IDH point mutation, is it an oncogenetic mechanism? Neuropathology 2017; 37: 582-585. https://doi.org/10.1111/neup.12401

[4] CHOI C, GANJI SK, DEBERARDINIS RJ, HATANPAA KJ, RAKHEJA D et al. 2-Hydroxyglutarate detection by magnetic resonance spectroscopy in IDH-mutated patients with gliomas. Nat Med 2012; 18: 624-629. https://doi.org/10.1038/ nm.2682

[5] MARDIS ER, DING L, DOOLING DJ, LARSON DE, MCLELLAN MD et al. Recurring mutations found by sequencing an acute myeloid leukemia genome. $\mathrm{N}$ Engl J Med 2009; 361: 1058-1066. https://doi.org/10.1056/NEJMoa0903840

[6] AMARY MF, BACSI K, MAGGIANI F, DAMATO S, HALAI D et al. IDH1 and IDH2 mutations are frequent events in central chondrosarcoma and central and periosteal chondromas but not in other mesenchymal tumours. J Pathol 2011; 224: 334-343. https://doi.org/10.1002/path.2913

[7] BORGER DR, TANABE KK, FAN KC, LOPEZ HU, FANTIN VR et al. Frequent Mutation of Isocitrate Dehydrogenase (IDH) 1 and IDH2 in Cholangiocarcinoma Identified Through Broad-Based Tumor Genotyping. Oncologist 2012; 17: 72-79. https://doi.org/10.1634/theoncologist.2011-0386

[8] CAIRNS RA, IQBAL J, LEMONNIER F, KUCUK C, DE LEVAL L et al. IDH2 mutations are frequent in angioimmunoblastic T-cell lymphoma. Blood 2012; 119: 1901-1903. https://doi.org/10.1182/blood-2011-11-391748

[9] BABAKOOHI S, LAPIDUS RG, FARAMAND R, SAUSVILLE EA, EMADI A. Comparative analysis of methods for detecting isocitrate dehydrogenase 1 and 2 mutations and their metabolic consequence, 2-hydroxyglutarate, in different neoplasms. Appl Immunohistochem Mol Morphol 2017; 25: 334-337. https://doi.org/10.1097/PAI.0000000000000342

[10] BAL D, GRYFF-KELLER A. 1H and 13C NMR study of 2-hydroxyglutaric acid and its lactone. Magnetic Resonance in Chemistry 2002; 40: 533-536. https://doi.org/10.1002/ mrc. 1053

[11] LEMONNIER F, CAIRNS RA, INOUE S, LI WY, DUPUY A et al. The IDH2 R172K mutation associated with angioimmunoblastic T-cell lymphoma produces $2 \mathrm{HG}$ in $\mathrm{T}$ cells and impacts lymphoid development. Proc Natl Acad Sci U S A 2016; 113: 15084-15089. https://doi.org/10.1073/ pnas.1617929114

[12] YE D, GUAN KL, XIONG Y. Metabolism, Activity, and Targeting of D- and L-2-Hydroxyglutarates. Trends Cancer 2018; 4: 151-165. https://doi.org/10.1016/j.trecan.2017.12.005

[13] VAN DEN BENT MJ, DUBBINK HJ, MARIE Y, BRANDES AA, TAPHOORN MJB et al. IDH1 and IDH2 mutations are prognostic but not predictive for outcome in anaplastic oligodendroglial tumors: A report of the European Organization for Research and Treatment of Cancer Brain Tumor Group. Clin Cancer Res 2010; 16: 1597-1604. https://doi. org/10.1158/1078-0432.CCR-09-2902 
[14] PICHUMANI K, MASHIMO T, BAEK HM, RATNAKAR J, MICKEY B et al. Conditions for 13C NMR detection of 2-hydroxyglutarate in tissue extracts from isocitrate dehydrogenase-mutated gliomas. Anal Biochem 2015; 481: 4-6. https://doi.org/10.1016/j.ab.2015.04.017

[15] RAKHEJA D, MITUI M, BORIACK RL, DEBERARDINIS RJ. Isocitrate dehydrogenase $1 / 2$ mutational analyses and 2-hydroxyglutarate measurements in Wilms tumors. Pediatr Blood Cancer 2011; 56: 379-383. https://doi.org/10.1002/ pbc. 22697

[16] STRUYS EA, JANSEN EEW, VERHOEVEN NM, JAKOBS C. Measurement of urinary D- and L-2-hydroxyglutarate enantiomers by stable-isotope-dilution liquid chromatography-tandem mass spectrometry after derivatization with diacetly-L-tartaric anhydride. Clin Chem 2004; 50: 1391-1395. https://doi.org/10.1373/clinchem.2004.033399
[17] RASHED MS, ABOUL-ENEIN HY, ALAMOUDI M, JAKOB M, AL-AHAIDEB LY et al. Chiral liquid chromatography tandem mass spectrometry in the determination of the configuration of glyceric acid in urine of patients with Dglyceric and L-glyceric acidurias. Biomed Chromatogr 2002; 16: 191-198. https://doi.org/10.1002/bmc.126

[18] COLLINS RRJ, PATEL K, PUTNAM WC, KAPUR P, RAKHEJA D. Oncometabolites: A new paradigm for oncology, metabolism, and the clinical laboratory. Clin Chem 2017; 63: 1812-1820. https://doi.org/10.1373/clinchem.2016.267666

[19] KALININA J, AHN J, DEVI NS, WANG L, LI Y et al. Selective detection of the $\mathrm{D}$-enantiomer of 2-hydroxyglutarate in the CSF of glioma patients with mutated isocitrate dehydrogenase. Clin Cancer Res 2016; 22: 6256-6265. https://doi. org/10.1158/1078-0432.CCR-15-2965 